\title{
Bilateral port-site metastases following laparoscopy \\ surgery for a stage 1B adenocarcinoma of uterine \\ cervix with negative lymph node
}

\author{
Yeun Joo Shin, Hae Nam Lee, Dae Woo Lee \\ Department of Obstetrics and Gynecology, Buchoen St. Mary Hospital, \\ Catholic University Medical College, Seoul, Korea
}

\section{Introduction}

Port-site metastasis following laparoscopic surgery for malignancy is being recognized as a potential complication. Most cases occur after laparoscopy for a pelvic mass that subsequently proved to be malignant or in the case of a disseminated intraperitoneal disease. The rare cases of port-site metastasis following laparoscopy for endometrial and cervical cancer have been associated with the presence of regional lymph node metastasis or disseminated disease in the peritoneal cavity. We present a case report of bilateral port-site metastases in the absence of spread beyond the primary tumor.

\section{Case presentation}

A 53 year-old woman presented in November 2015 with about $2 \mathrm{~cm}$ endo-cervical lesion. The mass was biopsied with pathology consistent with a moderatelydifferentiated invasive endocervical adenocarcinoma. There was a endocervical mass that encompassed the majority of the cervix but the vaginal fornices were initially noted to be free of tumor. The parametrium was also free of tumor on exam. The bladder and rectal mucosa were normal. She was clinically staged and determined to have invasive adenocarcinoma of the endocervix, FIGO Stage IB1. (Fig.1)

The patient underwent a laparoscopic radical hysterec tomy, bilateral salpingo-oophorectomy, pelvic and paraaortic lymph node dissection. In total, 33 pelvic and para-aortic lymph nodes were removed. Final pathology found all surgical margins and all lymph nodes to be negative for tumor. There was no lympho-vascular space invasion. Depth of cervical stromal invasion was $4 \mathrm{~mm}$. Width of invasion was $7.5 \mathrm{~mm}$. Based on these findings, the decision was made not to administer adjuvant therapy. During postoperative 1 year, she was intermittently monitored with physical exams, vaginal cytology every 3-6 months and imaging study. There were no evidences of clinical recurrence. In October 2016, during a routine follow-up visit, the patient reported palpable abdominal mass.

She underwent a CT scan of abdomen and pelvis, which revealed a new $5.8 * 5.7 * 5.3 \mathrm{~cm}^{3}$ sized para umbilical and $3.8 * 3.6 * 3.2 \mathrm{~cm}^{3}$ sized left paraumbilical mass in subcutaneous layer. Left internal pelvic lymph node metastases were visualized on PET-CT scan. (Fig. 2)

In November 2016, the patient underwent radical abdominal wall resection of the mass including metastatic left pelvic lymph node resection.
On final pathology, the tumor was confirmed to be metastatic cervical adenocarcinoma. Following diagnosis of the port site metastasis, the patient was treated with concurrent chemoradiotherapy.

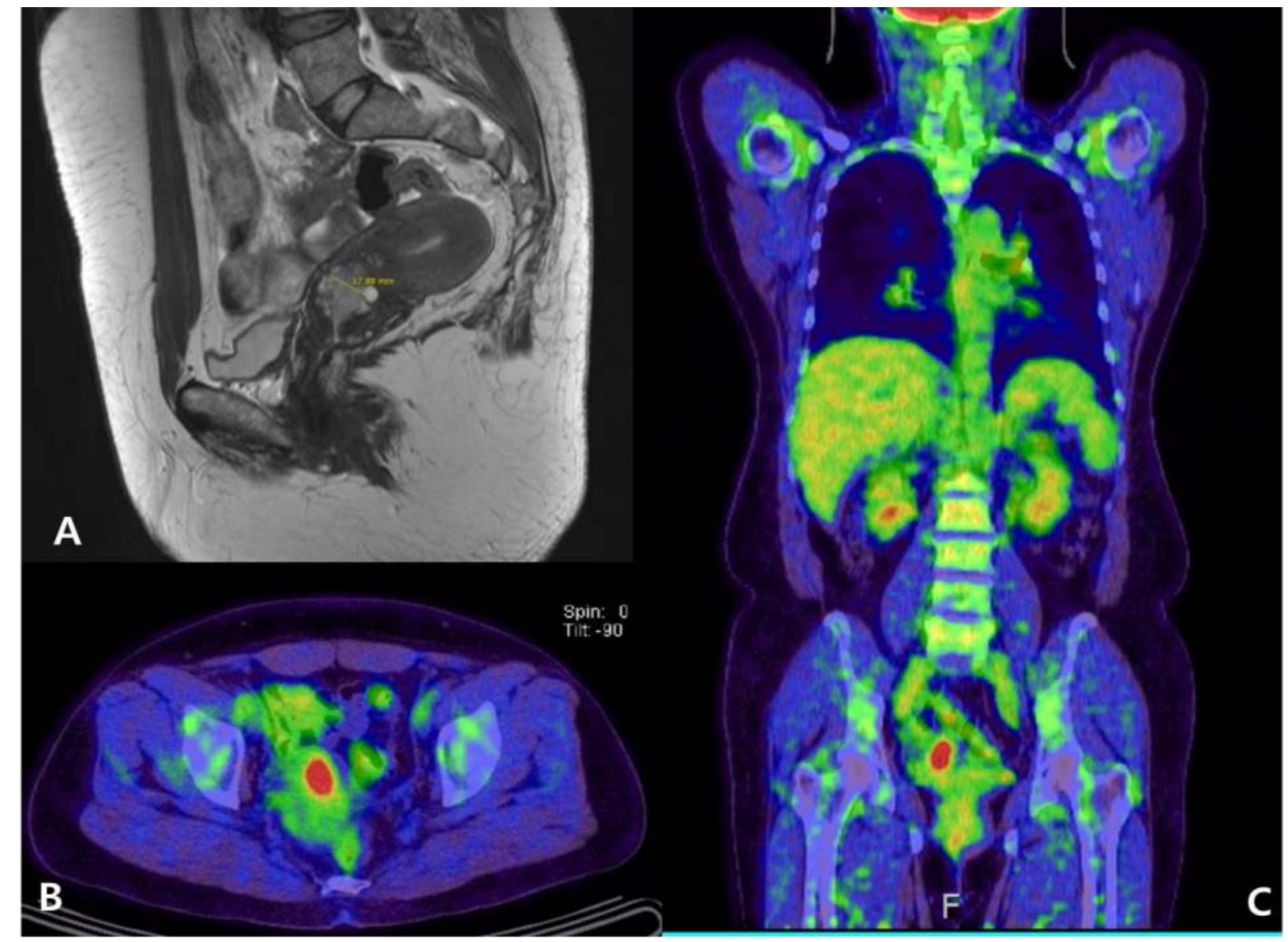

Fig. 1 Initial preoperative findings on MRI and PET-CT, $A: 2 \mathrm{~cm}$ sized $\mathrm{T} 2$ high signal intensity mass on endocervix B,C: Focal FDG uptake on uterine cervix

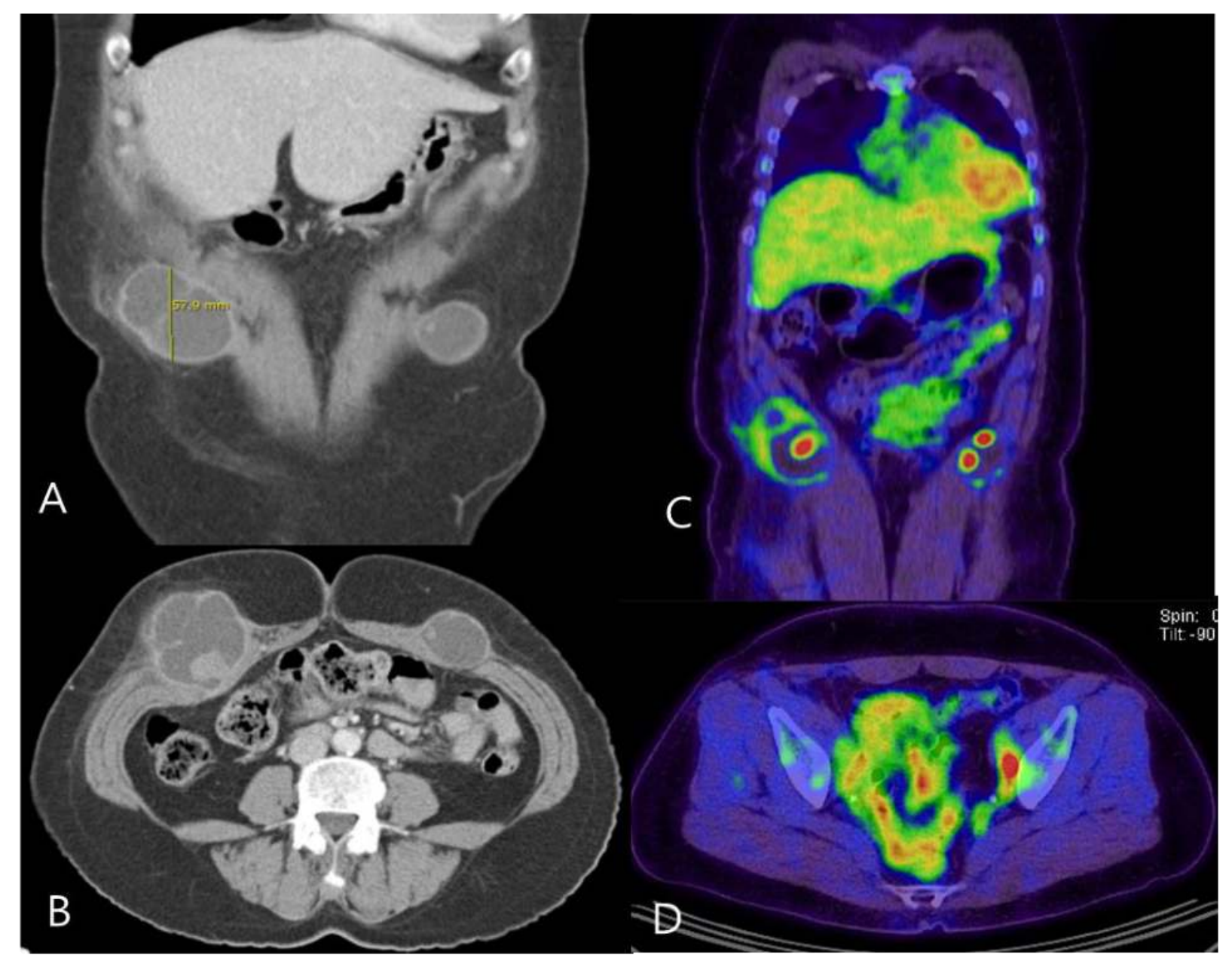

Fig. 2 Radiologic findings of abdominal wall port-site metastases, A,B,C: CT and PET-CT scan showing bilateral port-site mass, D: FDG uptake on left pelvic lymph node

\section{Conclusion}

This case demonstrates the potential for port-site metastases in patient who underwent laparoscopic surgery with negative surgical margins, negative lymph nodes, and absence of intraperitoneal disease. All patients should be closely monitored for recurrent disease with long-term follow-up, including special attention to the port-sites. 